\title{
The \\ DELPHI \\ Time Projection Chamber ${ }^{1}$
}

\author{
Y. Sacquin ${ }^{2}$
}

\begin{abstract}
The DELPHI central tracking device, a Time Projection Chamber, is described. Its performances in terms of resolution and particle identification are reviewed. Details on operation procedure, as calibration and distortion studies, are given.
\end{abstract}





\section{Description}

The Time Projection Chamber (TPC) is the central detector of the DELPHI experiment installed on the LEP collider at CERN. It consists of a cylindrical vessel of carbon fiber, built to stand vacuum, filled with a mixture of argon/methane $(80 \% / 20 \%)$, at atmospheric pressure. The vessel contains an electric cage consisting of copper strips $(3 \mathrm{~mm}$ wide, separated by 1 $\mathrm{mm}$ ) on a kapton foil, glued on both sides of two cylindrical epoxy skins defining the inner and the outer electric cages. The electric field is set through a central high voltage plane at $20 \mathrm{kV}$. A chain of 333 high precision $4 \mathrm{M} \Omega$ resistors connecting the copper strips defines a homogeneous field of $150 \mathrm{~V} / \mathrm{cm}$ over the whole drift volume.

Each end-cap of the TPC is divided in 6 sectors equipped with proportional multiwire chambers. Each chamber contains three planes of wires, a gate grid, a cathode wire plane defining the end of the drift area, and a sense plane of 192 wires (20 $\mu \mathrm{m}$ diameter), with a pitch of $4 \mathrm{~mm}$, interspersed with field wires. The anode sense wires are set to a high voltage of $1385 \mathrm{~V}$. A gap of $4 \mathrm{~mm}$ separates the sense wire plane from the rigid structure of the chamber covered by a cathode copper plate, on which are engraved 16 circular rows of capacitive pads. The surface of each pad is constant $\left(52.5 \mathrm{~mm}^{2}\right)$ and the number of pads per row (from 64 to 144) is kept a multiple of 16 for read-out convenience, which implies different pad widths. The rows are distant of $4.5 \mathrm{~cm}$, centered near the TPC axis and at 40 to $110 \mathrm{~cm}$ from this axis, as shown in figure [1]. There are 1680 pads per sector, and a total of 22464 channels to be read out.

The total drift length on each side of the central plane is $133 \mathrm{~cm}$. The whole TPC is located inside the DELPHI magnet which produces a homogeneous magnetic field of 1.2 tesla, parallel to the electric field in the backward region of the detector, antiparallel in the other part. The electrons are drifting in the gas with a velocity $\mathrm{V}_{d}=6.67 \mathrm{~cm} / \mu \mathrm{s}$ and a cyclotron frequency such that $\omega \tau=5.2$.

The pads and wires are read out by hybrid preamplifiers located inside the vessel, and their signals are sent, through $30 \mathrm{~m}$ long cables, to FASTBUS crates in the control room. The signal is first shaped gaussian with a $250 \mathrm{~ns}$ width, maintaining the integrated size of the signal. Then the signal is digitized through 13.5 MHz FADC (Thomson EF8305) with a broken linear characteristic allowing the 8 bit output to have a dynamic range equivalent to 9 bits. The same FASTBUS unit also performs zero-stripping and another FASTBUS crate processor (FIP) prepares the data to be read by the central TPC processor, controlled by the general data acquisition computer. The maximum drift time, $20 \mu \mathrm{s}$, corresponds to 270 FADC counts. Up to 4 events can be stored in the front-end buffers, with a dead time not exceeding the maximum drift time.

\section{Calibrations}

The calibration of the electronic chain proceeds through wire pulsing, radioactive sources and actual tracks. Laser tracks are used to monitor the drift velocity as well as to measure distortions.

The relative gain of the pads and wires is measured by pulsing the field wires with a positive signal (for wire gain) or negative signal (for pad gain). Different amplitudes of pulses are needed to cover the dynamic range and to determine the two slopes of the broken charac- 
teristic. This calibration is important for the $R \phi$ (pads) and $\mathrm{dE} / \mathrm{dx}$ (wires) measurement. It also provides a relative timing of each channel inside a sector, essential for the $z$-coordinate measurement.

Sources of ${ }^{55} \mathrm{Fe}$ are located in the sector end plates, on three radial lines of holes which can be remotely opened or closed. The determination of the position of the peak amplitude provides an absolute calibration of each wire on three points along it.

Actual tracks from $Z^{\circ}$ decays are used for relative sector to sector timing : for tracks leaving the TPC through the end-caps, the time when the the tracks cross the wire plane defines the zero time for the drift measurement. This determination defines the global wire time offset of each sector relative to the other ones. The time association of the hits on the pads and on the wires in each sector gives the relative time offset between pads and wires. From both measurement we get the relative sector to sector time offset of the pads.

The $45 \mathrm{GeV} / \mathrm{c}$ muon tracks from $Z^{\circ}$ decays are also used to cross-check the absolute normalisation of the wire gains, in clean conditions (isolated tracks). The reconstruction of vertices from hadronic tracks separately on the positive and negative $z$ sides is used for the absolute timing determination - the vertices having to be in the same $z$ position for the event - once the drift velocity is known.

Each sector is equipped with a laser for the drift velocity measurement. Each laser beam is divided and reflected, producing three tracks in front of each chamber, allowing for two separate measurements of the drift velocity. A careful study of the systematic error sources has been carried out. The present level of precision of this measurement, due to systematic errors, is better than $3 \times 10^{-4}$. The laser tracks have also been used to monitor the distortion changes induced by any modification of the parameters defining the electric field, as the values of the last two resistors or the voltage applied to the gate grid.

\section{Resolutions}

The amplitude and time of each hit is determined by using a parabolic adjustment on the three highest consecutive codes of the cluster. The space point determination is done assuming a gaussian shape of the signal over adjacent pads, imposing a width $\sigma$ to the response function. For points recorded with two pads, only one solution is possible. For three pad points, the weighted mean of the three possible combinations gives the better resolution. The width of the response function, $\sigma$, has been determined using clean pad points and parametrized as follows :

$$
\begin{aligned}
\sigma^{2}=\sigma_{0}^{2} & +k \cdot \delta \\
& +\sigma_{d}^{2} \cdot\left(1+\tan ^{2} \alpha_{w}\right) \cdot \cos ^{2}\left(\alpha_{w}-\alpha_{p}\right) \cdot L_{d} \\
& +\sigma_{w}^{2} \cdot\left(\tan \alpha_{w}-\tan \psi\right)^{2} \cdot \cos ^{2}\left(\alpha_{w}-\alpha_{p}\right) \\
& +\sigma_{p}^{2} \cdot \tan ^{2} \alpha_{p}
\end{aligned}
$$

In this formula, $\delta$ is the pad width associated to the row, which varies from 5.97 to 7.72 mm. $L_{d}$ is the drift distance, and we measure $\sigma_{d}=996 \mu m / \sqrt{ } m . \alpha_{w}$ and $\alpha_{p}$ are the angles of the tangent to the trajectory with the normal to the wire or pad row directions, respectively, in projection on the $x y$ plane. $\psi$ is the Lorentz angle, for which we find $\tan \psi=-0.68$. 
With this response function, the average residuals for tracks with $P_{t}$ greater than $1.5 \mathrm{GeV} / \mathrm{c}$ is found to be $230 \mu \mathrm{m}$ in $R \phi$, and $900 \mu \mathrm{m}$ in $z$. The resolution is better for 3 pad points only (40\% of all the points) : 220 and $880 \mu \mathrm{m}$.

Figure [2] shows the dependence of the residuals on $\alpha_{w}$. The minimum reached by the data $(190 \mu \mathrm{m})$ is above the minimum of the curve, which is at the Lorentz angle, $-34^{\circ}$, because tracks are limited to a range of $\pm 30^{\circ}$ in a sector. The dependence on $\alpha_{p}$ is shown in figure [3], where we observe a minimum around $3^{\circ}$. We note that all tracks with a $P_{t}$ above $2 \mathrm{GeV} / \mathrm{c}$ have a maximum angle $\alpha_{p}$ of about $5^{\circ}$, for which we are still in the lower part of the curve : this confirms the validity of the design of circular rows for an improved resolution of the energetic tracks.

Figure [4] shows the $z$ dependence of the $R \phi$ residuals, for 2 pad and 3 pad points. We see that for the small drift distances (large $z$ ) the difference between the two types is slight, since the transverse diffusion is small; at larger drift distances the improvement given by the 3 pad points is clear. This dependence should be taken into account when comparing the 3 pad from the 2 pad points contributions, since the 3 pad points are more frequent on the inner pad rows (due to smaller pad widths), which contribute mainly to the long drifts.

\section{Distortions}

During the alignment procedure, some track distortions have been seen, and first studied using the DELPHI Outer Detector (OD), which provides a fairly accurate measurement in $R \phi(\sigma \simeq 100 \mu \mathrm{m})$ at a large level arm $(2 \mathrm{~m})$. Using the muons from $Z^{\circ}$ decays, constrained to pass through the OD points and the mean beam position, the $R \phi$ deviations of the TPC have been determined and parametrized. Figure [5] shows those residuals, plotted as function of the pad row number. The difference between both half of the TPC results from a better adaptation, on one side, of the last resistors of the field cage. The figure shows an average over $\phi$, but the parametrisation is done for each sector. The dependence with $\theta$ was not extracted here because of the limited statistics.

For the $\theta$ dependence, and the $z$ distortions, hadronic tracks have been used. This was possible after laser track studies which have shown that the distortions were localized near the field cages. Hadronic tracks of transverse momentum greater than $2 \mathrm{GeV} / \mathrm{c}$ were refitted using the central pad rows only (rows 6 to 11), and residuals studied in the other rows. This leads to corrections in $R \phi$ and $z$, with a dependence of those corrections with $\theta$.

The distortions have been traced to come from the exact adaptation of the last resistors of the degraded potential chain, which should match the positioning of the cathode wire plane defining the end of the drift field. Moreover the gate grid has to be set to a potential which minimizes the distortions.

\section{$5 \mathrm{dE} / \mathrm{dx}$ determination}

The amplitudes of the charges deposited by each track on up to 192 wires are used to estimate the energy loss of the particle, using as an estimator the mean value of the $80 \%$ lowest amplitudes associated to the track. Several corrections, in addition to standard calibrations, have to be applied to this estimator : 
- normalisation to $4 \mathrm{~mm}$ path (angle correction);

- residual gap correction, to correct for the bias induced by the truncation procedure ;

- drift distance correction ; this has to be applied only if the maximum amplitudes of the clusters are used ; since the shapers keep the integrated amplitudes, no correction is done when they are used ;

- track separation : only the hits which are separated in $z$ by at least $2 \mathrm{~cm}$ are considered ; this reduces the number of hits in hadronic event tracks : $65 \%$ of the tracks have more than 40 isolated hits, and $35 \%$ more than 100 hits ;

- sector edge effect : a loss of gain is observed for points near the radial edges of the sectors ; this has been parametrized and corrected for ;

- accumulated charge effect : due to an inadequacy in the electronics design, an oscillation following the signals affects the later hits on the same channel ; this has been parametrized and corrected for, and an upgrade of the electronic is in progress.

With the above corrections, the resolution of the $\mathrm{dE} / \mathrm{dx}$ measurement for minimum ionizing particles is $6.2 \%$, and for electrons $5.7 \%$, as shown in figure [6]. The separation between $\pi$ and $e$ is effective up to $5 \mathrm{GeV} / \mathrm{c}$ momentum, and substantial progress is expected. The $\mathrm{dE} / \mathrm{dx}$ measurement of the TPC is successfully used in physics analysis, to enhance inclusive signals.

\section{Conclusions}

The DELPHI TPC project shows that the idea of circular pad rows is a success. The operation of a large TPC needs careful monitoring and calibrations. Tiny causes make distortions almost unavoidable. The nominal performances in terms of resolution and $\mathrm{dE} / \mathrm{dx}$ have been reached, and work is continuing to improve them.

The TPC group ${ }^{1}$ thanks the many engineers and technicians for their work and skill during the construction and operation of the TPC. They have been providing DELPHI with an outstanding central detector since the first LEP beams.

${ }^{1}$ DAPNIA/SPP, CEN Saclay, F ; College de France, Paris, F ; LAL, Orsay, F ; CERN, CH ; Lund University, Lund, $S$. 

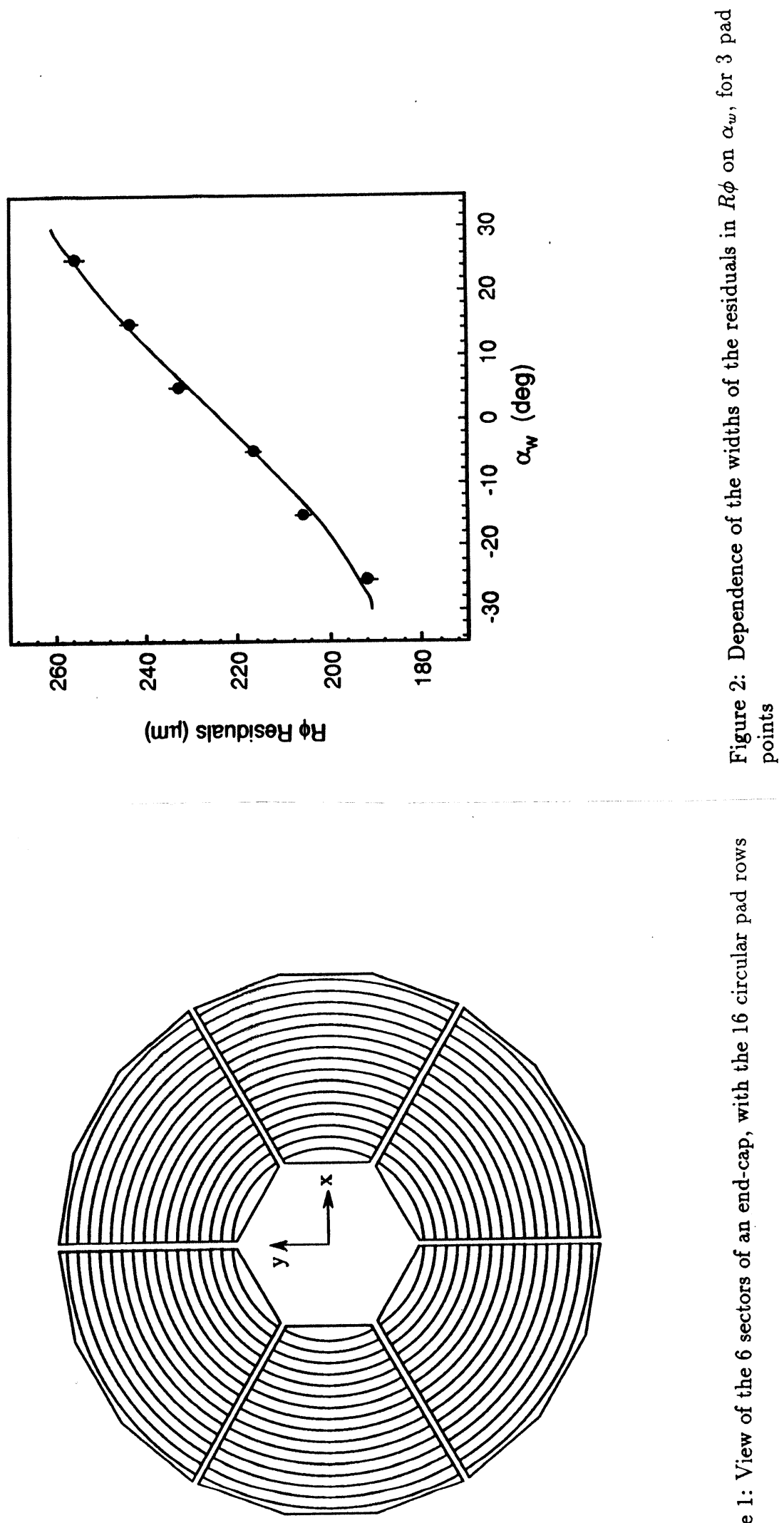

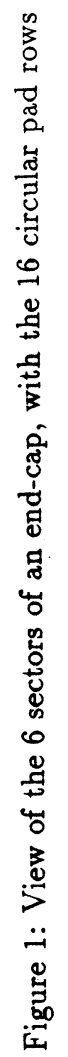



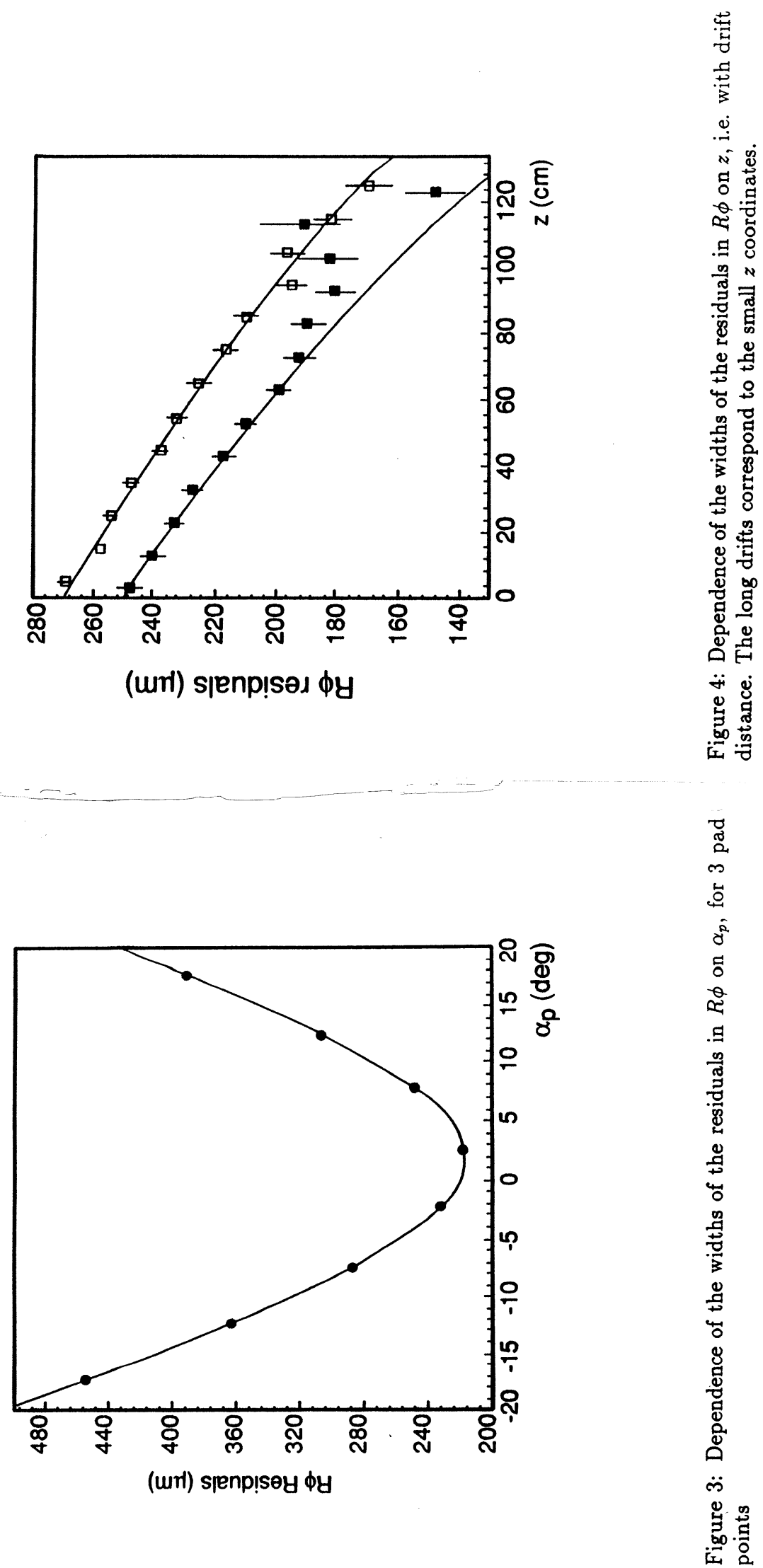

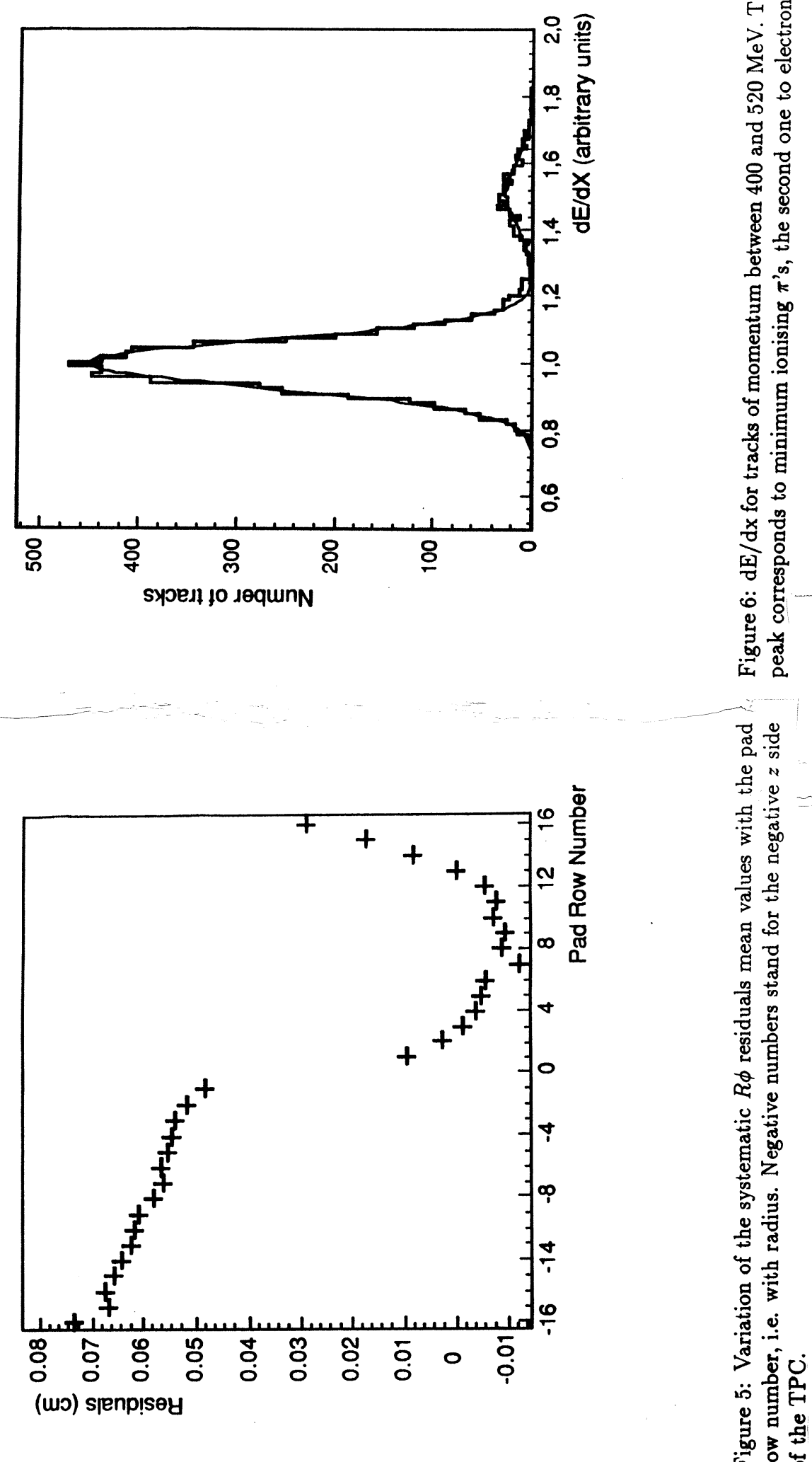